\section{Prophylaxe durch Pertussisimpfstoff?}

Die Bildung von IgE-Antikörpern als Immunantwort auf manche Impfungen ist bei Atopie stärker ausgeprägt. Sie kann aber, wie vorangegangene Studien zeigen konnten, durch eine gleichzeitige Pertussisimpfung modifiziert werden. Ob dies lediglich für den zellulären oder aber auch für den nebenwirkungsärmeren und heute üblicheren azellulären Impfstoff gilt, untersuchte eine Arbeitsgruppe der Charité in Berlin. Sie verglich die verschiedenen Impfungen bezüglich der ausgelösten IgE- und IgG4-Antwort auf die gleichzeitig verabreichten Diphtherie- und Tetanusantigene.

Untersucht wurden insgesamt 265 Kinder, die dreimal entweder nur gegen Diphtherie und Tetanus oder zusätzlich mit zellulärem bzw. azellulärem 2- oder 5-Komponenten-Impfstoff gegen Perrussis geimpft wurden. Im Alter von 2, 7 und 12 Monaten wurden mittels RAST die Werte von IgE und IgG4 gegen Diphtherie und Tetanusantigene bestimmt.

Vor der ersten Impfung wurden die entsprechenden Immunglobuline nur in einem Fall gefunden. Einen Monat nach Abschluss der Impfungen waren sowohl die IgE- als auch die IgG4-Antikörper gegen Diphtherie und Tetanus bei den Kindern ohne Keuchhustenimpfung und bei azellulär geimpften deutlich häufiger nachweisbar als bei Kindern, die den zellulären Impfstoff erhalten hatten $(p<0,001)$. Dies galt für Kinder mit oder ohne atopische Veranlagung.

Es bestätigt sich also, dass die ganzzelluläre Pertussisimpfung die Bildung von IgE und $\mathrm{IgG}_{4}$ gegen mitgeimpfte Antigene herunterreguliert. Der Effekt ist vermutlich auf Zellwandbestandteile von Bordetella pertussis zurückzuführen. Die vorliegenden Ergebnisse könnten ein erster Schritt dahin sein, mittels Impfung die Bildung von IgE auch gegen weitere Umweltantigene zu verhindern. Ob damit auch klinisch die Ausbildung von Allergien beeinflusst werden kann, ist noch unklar. ib

Grüber C et al. Suppression von IgE gegen Tetanus- und Diphtherie-Toxoid bei Kindern nach ganzzellulärer (aber nicht azellulärer) Bordetella-pertussisImpfung. 16. Mainzer Allergie-Workshop, Mainz, 12.-13. März 2004 unabhängige und kostengünstige Verbreitung von Informationen in gleicher Weise sicherstellen. „Natürlich“, so Erdmann, „wohnen in meiner Brust zwei Seelen: Auch ich möchte schnellen $\mathrm{Zu}$ gang zu vielen Dingen. Aber man muss hier einen Mittelweg finden."

\section{Enteignung von Verlagen und Autoren}

Wie der Justiziar des Börsenvereins des Deutschen Buchhandels Dr. Christian Sprang, Frankfurt, darstellte, muss die deutsche Politik zurzeit das Problem lösen, trotz der bestehenden Finanzkrise weiterhin den freien und ungehinderten Zugang zu wichtigen Informationen zu ermöglichen. Die Änderungen des Urheberrechtsgesetzes und die Einführung von Open-access-Publikationen erscheinen ihm schädlich für Wissenschaft und Gesellschaft. Das von der VG-Wort bezahlte Entgelt für die Nutzung publizierter Artikel und Buchkapitel im Sinne des $\$ 52 \mathrm{a}$ ist lediglich ein geringer Obolus - von der Bezahlung von Marktpreisen kann dabei nicht die Rede sein. „Geistiges Eigentum sieht man nicht. Einen PC würde so leicht niemand stehlen", formulierte Sprang provokativ. Nach seiner Ansicht werden Verlage und Autoren durch die Regelungen tatsächlich enteignet: Je mehr Leser ihre Zeitschriften abbestellen, weil sie anderweitig an das publizierte Wissen gelangen, um so teurer wird das Abonnement - ein Teufelskreis. Eine der wichtigsten Aufgabe der Verlage ist die Auswahl, qualitative Aufbereitung und Verbreitung von Informationen. Die bereits eingeführten und die geplanten Änderungen am UrhG haben nach Sprangs Meinung letztlich zur Folge, dass privates Kapital aus dem Bereich der Wissenspublikation abgezogen werden wird und der Staat diese Aufgabe lösen muss. „Der Staat kann dies aber nicht besser und billiger - es wird nur schlechter und teurer werden."

Das Urheberrecht zu entwerten ist nach Sprangs Meinung ein Fehler, sein Fazit: Internet und Schnelligkeit nutzen ja, aber nur in derselben Qualität wie bisher - nach kritischer Vorauswahl und redaktioneller Aufbereitung.

Die beiden Protagonisten einer Änderung des Copyrights, Gabriele Beger von der Zentral- und Landesbibliothek Berlin, und Ulrich Korwitz, Direktor der Deutschen Zentralbibliothek für Medizin in Köln, bemühten sich redlich, die Harmlosigkeit des Zugriffs auf das geistige Eigentum der Autoren bzw. der Verlage darzulegen. Selbstverständlich würden Verlage honoriert, wenn eine Bibliothek Publikationen dieses Verlages online stellt. Die Frage, ob denn diese Honorierung angemessen sei, blieb jedoch offen.

Auch war nicht klärbar, ob bei Open-access-Publikationen nicht die Autoren letztendlich die Zeche zahlen: Zunächst verzichten sie auf ein etabliertes, mit entsprechendem Impact versehenes Journal, um im weitgehend anonymen Internet $\mathrm{zu}$ veröffentlichen, dann müssen sie dafür auch noch zahlen. Daran wird sich auch nicht viel ändern, wenn man - ganz gegen die Usancen bei den etablierten Verlagen - den Mitgliedern des Reviewing-Boards auch noch Honorare zahlt. So blieben bisher, wie sich auf eine Diskussionsfrage hin ergab, die mit Geldern der wissenschaftlichen Fachgesellschaften geförderten Online-Journals noch weitgehend ohne echte Originalbeiträge.

\section{Keine Qualität ohne Kosten}

Insofern ist zu fürchten, wie Erdmann nochmals zu bedenken gab, ob nicht die ganze Kampagne gegen die Verlage bloß Gelder verschlingt, die sich besser in der klinischen Forschung verwenden ließen.

Die Wissensgesellschaft, so Wallwieners Resümee, braucht die schnelle und günstige Verbreitung von Informationen. Wie kostengünstig dies möglich sein wird, bleibt abzuwarten: „Qualität ist immer teuer". Andererseits will niemand den Autor um sein geistiges Eigentum bringen. Open Access muss daher reglementiert werden: Das Urheberrecht bleibt beim Autor, Geldgeber und Nutzer regulieren die Qualität. Dr. Ute Mader, Stuttgart Dr. Till U. Keil, München

Verlagssymposium Thieme und Urban \& Vogel „Das Urheberrecht: Was geschieht in Deutschland mit dem geistigen Eigentum der Autoren?". 110. Tagung der Deutschen Gesellschaft für Innere Medizin, Wiesbaden, 17.-21. April 2004 